\title{
A huge recurrent retroperitoneal inflammatory myofibroblastic tumor implicating renal and ureter: a case report
}

\author{
Tianle Zhu", Pan Gao", Jingjing Gao, Xi Liu, Xiansheng Zhang \\ Department of Urology, The First Affiliated Hospital of Anhui Medical University, Hefei, China \\ \#These authors contributed equally to this work. \\ Correspondence to: Xiansheng Zhang. The First Affiliated Hospital of Anhui Medical University, Hefei, China. Email: xiansheng-zhang@163.com.
}

\begin{abstract}
Inflammatory myofibroblastic tumor (IMT) is a rare sarcoma with unique molecular characteristics. It also has typical fasciitis-like, dense spindle cells and hypocellular fibrous histologic patterns and can occur at any age. The etiology of IMT is unknown and a complete treatment and monitoring plan for IMT have not been developed. We have found a rare case about a huge recurrent IMT implicating renal and ureter. The patient was accompanied by a history of a lesion sized $15.5 \mathrm{~cm} \times 11.5 \mathrm{~cm} \times 10.5 \mathrm{~cm}$ as a retroperitoneal IMT, and the first tumor resection was performed 12 months ago. The patient, who suffered from tumor recurrence within 1 year and whose recurrent tumor also invaded into the renal parenchymal was given a retroperitoneal neoplasm resection plus right nephrectomy. In order to clarify the pathological features, the histological specimens of recurrent retroperitoneal IMT were examined as well as primary retroperitoneal IMT, and immunohistochemical (IHC) stains showed that the spindle cells were positive for vimentin and negative for anaplastic lymphoma kinase (ALK). At 24 months follow-up, the patient recovered well and there was no evidence of tumor recurrence. This case report is conducive to understanding this rare tumor. When conditions permit, complete surgical resection is the recommended treatment for primary and recurrent IMT.
\end{abstract}

Keywords: Inflammatory myofibroblastic tumor (IMT); renal; retroperitoneal inflammatory myofibroblastic tumor; case report

Submitted May 24, 2021. Accepted for publication Jul 30, 2021.

doi: $10.21037 /$ tcr-21-899

View this article at: https://dx.doi.org/10.21037/tcr-21-899

\section{Introduction}

Inflammatory myofibroblastic tumor (IMT) is a lesion composed of myofibroblasts and fibroblasts with the inflammatory infiltrates of plasma cells, lymphocytes, and eosinophils (1-5). The pathogenesis of IMT is controversial (6-9). It is similar to a benign tumor in manner of spindlecell proliferation intermixed with inflammatory cells, which occurs in a wide variety of body sites, including brain, lung, genitourinary tract, abdominal cavity, mesentery, bladder etc. $(3,10)$. Recent researches showed that the characterization of IMT is a neoplasm with the potential for local aggressiveness, recurrence, metastasis, and malignant transformation (6). IMT is so rare that its incidence is not clear. So far, only a few cases have been reported in the literature. The symptoms of IMT, which are not specific, usually vary in the different locations of the tumor (11), so it is difficult to diagnose. At present, the common methods for diagnosing IMT are ultrasound, abdominal computed tomography (CT) or magnetic resonance imaging $(12,13)$. The radical resection is the main treatment for patients with IMT (14). IMT often develops in the lung of children and young adults but seldom occurs in older patients (12). The retroperitoneum is also an unusual site of presentation for the rare tumor. In the paper, a case of recurrent retroperitoneal IMT in a 62-year-old woman is reported and the clinical and pathological features are discussed with the diagnosis of this rare tumor. We present the following case in accordance with the CARE reporting checklist (available at https://dx.doi.org/10.21037/tcr-21-899). 

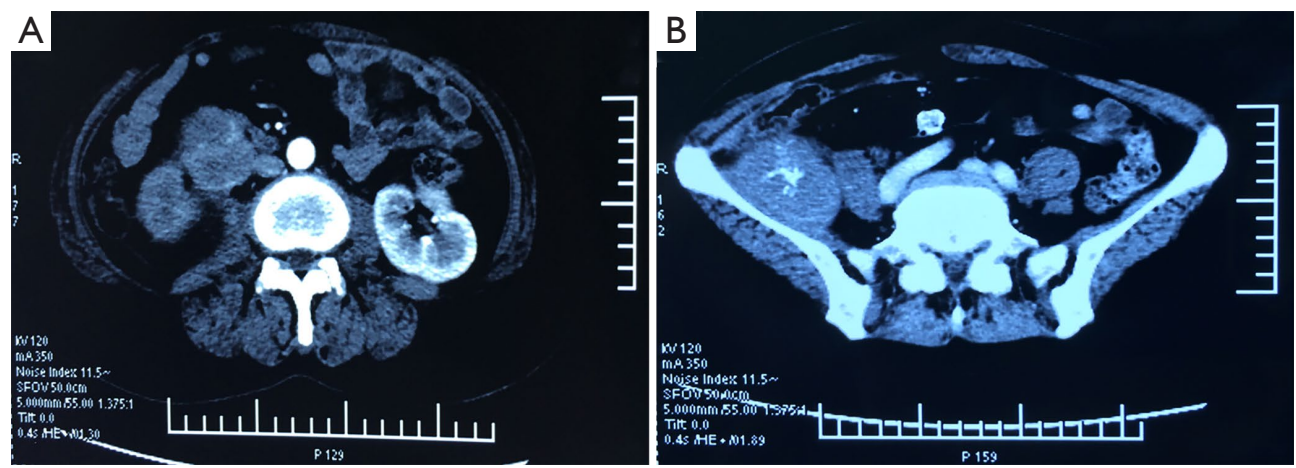

Figure 1 Contrast-enhanced CT of abdominal and pelvic. (A) Contrast-enhanced CT showed a $7 \mathrm{~cm} \times 5 \mathrm{~cm}$ irregular lump conspicuously located in the right ureter. (B) Contrast-enhanced CT showed a $6 \mathrm{~cm} \times 5 \mathrm{~cm}$ irregular lump located in the right iliac fossa. CT, computed tomography.

\section{Case presentation}

A 62-year-old woman suffered from flank pain without apparent causes 2 months, with no evidence of fever or hematuria. The flank pain was aggravated when a right lower abdominal mass was palpated. She was admitted to the Second People's Hospital of Anhui Province in February 2019. Abdomen ultrasonic B-scan showed a solid lesion under the right renal, which indicated retroperitoneal lesions. For further treatment, she was admitted to our hospital on March 2, 2019. She was accompanied by a history of a lesion sized $15.5 \mathrm{~cm} \times 11.5 \mathrm{~cm} \times 10.5 \mathrm{~cm}$ as a retroperitoneal IMT with retroperitoneal tumor resection 1 year ago.

Laboratory tests showed moderate anemia, normal urine tests, and normal tumor markers. Ultrasonography revealed a hypoechoic, heterogeneous solid mass located beneath the lower pole of the right kidney, which was closely associated with the kidney. The diameter of right-side upper ureter was dilated to $14 \mathrm{~mm}$ accompanied with the right hydronephrosis due to the mass pressed the ureter. It also showed a mild spleen swelling. Further tests, including abdominal and pelvic enhanced CT scan, revealed a $7 \mathrm{~cm}$ $\times 5 \mathrm{~cm}$ irregular lump conspicuously located in the right ureter (Figure $1 A$ ), and a $6 \mathrm{~cm} \times 5 \mathrm{~cm}$ irregular lump located in the right iliac fossa (Figure 1B), respectively.

Two weeks after the admission, we performed retroperitoneal tumor resection and right nephrectomy to clarify the pathological features of the lesion.

Histologically, the lesion sized $19 \mathrm{~cm} \times 7 \mathrm{~cm} \times 3 \mathrm{~cm}$, which invaded into renal parenchyma, did not intrude into ureter cut edge (Figure 2A,2B). Having been cutting, the lesion showed the cystic mass, including pale yellow liquid. Renal parenchyma was atrophied, and renal cortex was unclear. The lesion consisted of a major of spindle cells forming beam shape. Partial cells showed irregular cell form, which included mononuclear, multinuclear and ganglion-like cells (Figure 2C). Mesenchyme of the lesion showed lymphocyte and plasmacyte invasion. The necrosis and bleeding were present in some parts. Besides, evidence was obtained from the last year pathological and immunohistochemical (IHC) results of the patient (pathological and IHC tests were conducted from the last year tumor resection samples of the patient). The histological (Figure $3 A$ ) and IHC stains illustrated that the spindle cells were positive for vimentin. Positively stating of smooth muscle actin (SMA) (Figure 3B), P53 or Ki-67 was a small proportion. However, IHC evaluation revealed spindle cells to be negative for anaplastic lymphoma kinase (ALK) (Figure 3C), desmin, S-100 protein, creatinine kinase, Dog-1, CD117, CD34, CD68.

The operation of this patient went smoothly, and some pictures during the operation are displayed in Figure 4. According to the Clavien-Dindo classification system (15), the patient had minor postoperative complications (grade I). After postoperative, the right lower extremity numbness and paresthesia appeared. The patient was treated with mecobalamin, neurotropin and mouse Nerve Growth Factor so that the symptom disappeared. Patient had untypical/irregular fever with non-infection and was treated with aspirin-DL-lysine, after which the body temperature gradually recovered normal. The patient did not complain of any discomfort. A close follow-up was carried out. The patient remained healthy and fit without any uncomfortable feelings. We did not find any neonatal lesions from the 

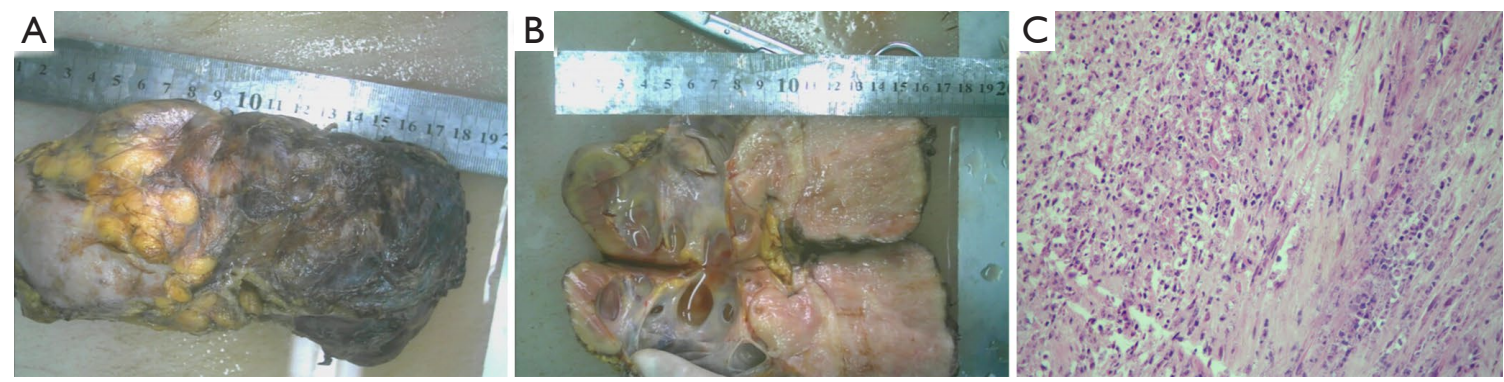

Figure 2 Morphology of recurrent retroperitoneal IMT and HE staining (400×). (A,B) Tumor invaded into renal parenchymal. (C) Irregular cell form including macronucleus, multinuclear and ganglion-like cells. IMT, inflammatory myofibroblastic tumor; HE, hematoxylin-eosin.
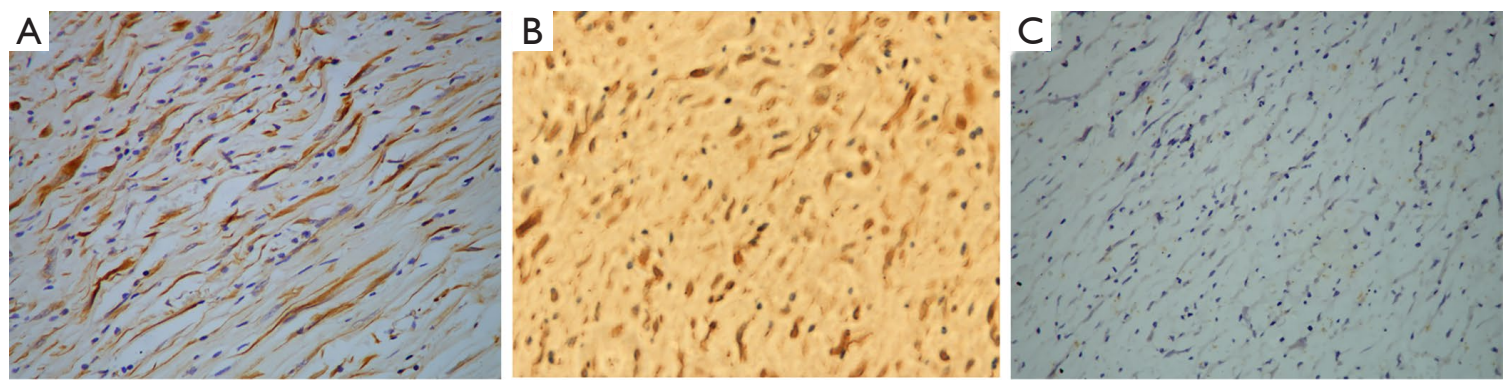

Figure 3 Morphology of recurrent retroperitoneal IMT by IHC stain. IHC staining for vimentin (A: 400×), SMA (B: 400×) and ALK (C: 400x). IMT, inflammatory myofibroblastic tumor; IHC, immunohistochemical; SMA, smooth muscle actin; ALK, anaplastic lymphoma kinase.
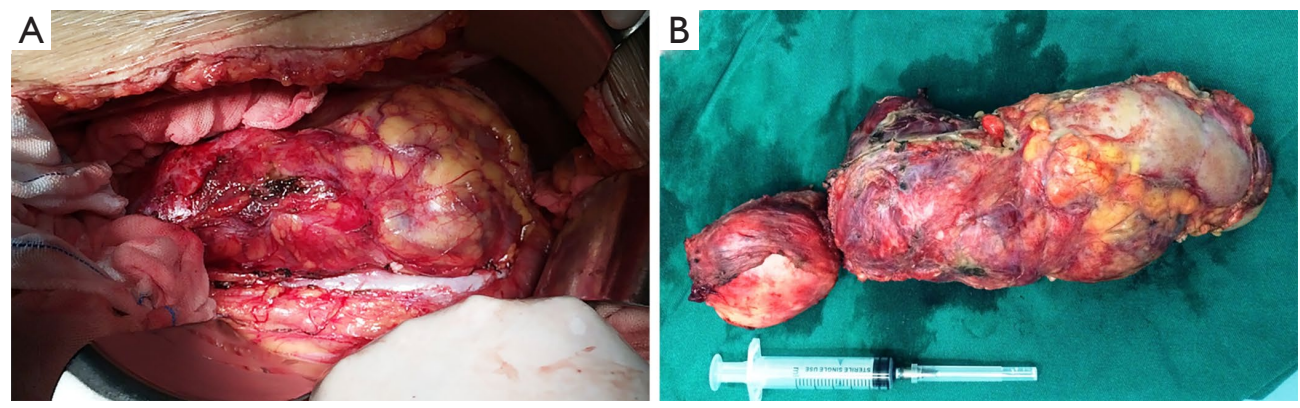

Figure 4 Photos of intra-operative findings. (A) A huge recurrent retroperitoneal tumor. (B) Tumor that was removed by surgery.

radiologic examination compared with the former one. All procedures performed in the studies involving human participants were in accordance with the ethical standards of the institutional and/or national research committee(s) and with the Helsinki Declaration (as revised in 2013). Written informed consent was obtained from the patient for publication of this case report and accompanying images. A copy of the written consent is available for review by the editorial office of this journal.

\section{Discussion}

The radiologic features showed that malignant IMT usually appears as a heterogeneous mass, which include a mix of solid and cystic components and infiltrative patterns simulating other aggressive malignant lesions on imaging (16). Due to the nonspecific symptoms and variable imaging features, it is difficult to make a definitive clinical diagnosis. In our case, ultrasonography showed 
a hypoechoic, inhomogeneous solid mass closely related to the kidney at the lower pole of the right kidney. The abdominal and pelvic enhanced CT scan also revealed that a huge unclear border mass affected the right kidney and the right ureter. This first clinic impression was liposarcoma because of the similar features. However, combining the patient's clinical symptoms, the history of IMT tumor resection and imaging examination, we seriously suspected that this case is recurrent retroperitoneal IMT.

In general, biopsy and postoperative histopathological analysis are helpful in the diagnosis of IMT $(13,17)$. On account of the rare and non-specific symptoms of IMT, clinicians hardly diagnose IMT before surgery or biopsy. Histologically, the recognized basic feature of IMT is mesenchymal spindle cell proliferation, which is accompanied by a myxoid background where reactive lymphocyte/plasmacyte infiltrate (18). Three histologic patterns have been described which could variably exist within the same tumor: a fascicular pattern, a hyalinized pattern and a fibrous hypocellular pattern $(2,19)$. In our case, the pathologic diagnosis of IMT is challenging as well. It is often difficult to differentiate the different members within the broad category of inflammatory pseudotumor owing to the overlapping histologic features.

The expression of ALK shown in IMTs makes it a distinct entity when IHC and molecular characteristics are considered $(20,21)$. The expression of ALK in IMTs also makes ALK a hallmark for the diagnosis of IMTs. The IHC profile of myofibroblasts make it possible for diagnosing IMT $(22,23)$. In our case, since the patient underwent radical retroperitoneal IMT resection a year ago and had problems such as tissue adhesion in the abdominal cavity, so according to the situation at that time we did not perform a preoperative biopsy on the patient. The results of IHC examination after operation played a supportive role in confirming IMT. However, in this case, the reason why the ALK was absent may be that this marker is more commonly expressed in younger patients $(1,24)$.

The recurrence rate of IMT varies from less than $2 \%$ of lung tumors to $25 \%$ of extrapulmonary lesions according to the anatomic site of development $(1,25)$. It occurs predominantly in the visceral soft tissue of children with a potential for local recurrence and rare distant metastasis (5). It is rare for adults in their 60 s to have a huge recurrent IMT that infiltrates into the right kidney and oppresses the right ureter only 1 year after surgery. The recurrent retroperitoneal IMT may be caused by uncompleted surgery resection. So far, due to the rarity of IMT, no complete treatment and monitoring protocol has been established. The role of radiotherapy and chemotherapy in IMT is unclear, but in unresectable cases, radiotherapy and chemotherapy may have potential benefits $(8,26,27)$. Surgical resection is still recommended for the treatment of recurrent lesions. In our case, after the patient was discharged from the hospital, we closely followed up for 24 months, and there was no tumor recurrence. However, this follow-up period is relatively short and vigilant monitoring is still needed to quickly detect further recurrence.

In conclusion, recurrent retroperitoneal IMT is very rare, especially in elderly patient who was performed primary IMT resection 1 year ago, which has not been reported before. The case report can enrich doctors' understanding of it. In addition, Preoperative imaging examination, biopsy and postoperative histological analysis are very helpful to determine the diagnosis of IMT. At present, surgical resection is the main treatment for most patients with IMT, and in order to prevent the recurrence of IMT, close monitoring must be carried out after operation.

\section{Acknowledgments}

We thank our anonymous reviewers for their valuable comments, which greatly improved our article. We also would like to thank Dr. Shengwei Ke for his help in polishing our paper.

Funding: None.

\section{Footnote}

Reporting Checklist: The authors have completed the CARE reporting checklist. Available at https://dx.doi. org/10.21037/tcr-21-899

Peer Review File: Available at https://dx.doi.org/10.21037/ tcr-21-899

Conflicts of Interest: All authors have completed the ICMJE uniform disclosure form (available at https://dx.doi. org/10.21037/tcr-21-899). The authors have no conflicts of interest to declare.

Ethical Statement: The authors are accountable for all aspects of the work in ensuring that questions related to the accuracy or integrity of any part of the work are appropriately investigated and resolved. All procedures 
performed in studies involving human participants were in accordance with the ethical standards of the institutional and/or national research committee(s) and with the Helsinki Declaration (as revised in 2013). Written informed consent was obtained from the patient for publication of this case report and accompanying images. A copy of the written consent is available for review by the editorial office of this journal.

Open Access Statement: This is an Open Access article distributed in accordance with the Creative Commons Attribution-NonCommercial-NoDerivs 4.0 International License (CC BY-NC-ND 4.0), which permits the noncommercial replication and distribution of the article with the strict proviso that no changes or edits are made and the original work is properly cited (including links to both the formal publication through the relevant DOI and the license). See: https://creativecommons.org/licenses/by-nc-nd/4.0/.

\section{References}

1. Fraggetta F, Doglioni C, Scollo P, et al. Uterine inflammatory myofibroblastic tumor in a 10-year-old girl presenting as polypoid mass. J Clin Oncol 2015;33:e7-e10.

2. Rabban JT, Zaloudek CJ, Shekitka KM, et al. Inflammatory myofibroblastic tumor of the uterus: a clinicopathologic study of 6 cases emphasizing distinction from aggressive mesenchymal tumors. Am J Surg Pathol 2005;29:1348-55.

3. Freeman A, Geddes N, Munson P, et al. Anaplastic lymphoma kinase (ALK 1) staining and molecular analysis in inflammatory myofibroblastic tumours of the bladder: a preliminary clinicopathological study of nine cases and review of the literature. Mod Pathol 2004;17:765-71.

4. Bahadori M, Liebow AA. Plasma cell granulomas of the lung. Cancer 1973;31:191-208.

5. Lai LM, McCarville MB, Kirby P, et al. Shedding light on inflammatory pseudotumor in children: spotlight on inflammatory myofibroblastic tumor. Pediatr Radiol 2015;45:1738-52.

6. Liu C, Zhao X, Zhao Z, et al. Malignant inflammatory myofibroblastic tumor of the prostate. J Clin Oncol 2013;31:e144-7.

7. Brittig F, Ajtay E, Jaksó P, et al. Follicular dendritic reticulum cell tumor mimicking inflammatory pseudotumor of the spleen. Pathol Oncol Res 2004;10:57-60.

8. Tao YL, Wang ZJ, Han JG, et al. Inflammatory myofibroblastic tumor successfully treated with chemotherapy and nonsteroidals: a case report. World J Gastroenterol 2012;18:7100-3.

9. Xiang J, Liu X, Wu S, et al. Multiple inflammatory myofibroblastic tumor of the duodenum: case report and literature review. J Gastrointest Surg 2012;16:1442-5.

10. Hayashi M, Kawakubo H, Mayanagi S, et al. Gastric inflammatory myofibroblastic tumor treated with combined laparoscopic and endoscopic gastric wedge resection: a case report. World J Surg Oncol 2018;16:161.

11. Gleason BC, Hornick JL. Inflammatory myofibroblastic tumours: where are we now? J Clin Pathol 2008;61:428-37.

12. Wu S, Chen L, Wan Q, et al. Inflammatory myofibroblastic tumor of the urinary bladder in a patient with the left renal cell carcinoma: A case report. Exp Ther Med 2014;7:1010-2.

13. Choi EJ, Jin GY, Chung MJ, et al. Primary Inflammatory Myofibroblastic Tumors of the Breast with Metastasis: Radiographic and Histopathologic Predictive Factors. J Breast Cancer 2015;18:200-5.

14. Fabre D, Fadel E, Singhal S, et al. Complete resection of pulmonary inflammatory pseudotumors has excellent long-term prognosis. J Thorac Cardiovasc Surg 2009;137:435-40.

15. Slaman AE, Lagarde SM, Gisbertz SS, et al. A Quantified Scoring System for Postoperative Complication Severity Compared to the Clavien-Dindo Classification. Dig Surg 2015;32:361-6.

16. Rasalkar DD, Chu WC, To KF, et al. Radiological appearance of inflammatory myofibroblastic tumour. Pediatr Blood Cancer 2010;54:1029-31.

17. Pan $\mathrm{Y}, \mathrm{Wu} \mathrm{X}, \mathrm{Liu} \mathrm{J}$, et al. Abnormal liver function induced by myofibroblastic sarcoma infiltrating the liver: A case report. Oncol Lett 2015;9:798-800.

18. Kovach SJ, Fischer AC, Katzman PJ, et al. Inflammatory myofibroblastic tumors. J Surg Oncol 2006;94:385-91.

19. Coffin CM, Hornick JL, Fletcher CD. Inflammatory myofibroblastic tumor: comparison of clinicopathologic, histologic, and immunohistochemical features including ALK expression in atypical and aggressive cases. Am J Surg Pathol 2007;31:509-20.

20. Teoh JY, Chan NH, Cheung HY, et al. Inflammatory myofibroblastic tumors of the urinary bladder: a systematic review. Urology 2014;84:503-8.

21. Cessna MH, Zhou H, Sanger WG, et al. Expression of ALK1 and p 80 in inflammatory myofibroblastic tumor and its mesenchymal mimics: a study of 135 cases. Mod Pathol 2002;15:931-8. 
22. Qiu X, Montgomery E, Sun B. Inflammatory myofibroblastic tumor and low-grade myofibroblastic sarcoma: a comparative study of clinicopathologic features and further observations on the immunohistochemical profile of myofibroblasts. Hum Pathol 2008;39:846-56.

23. Farris AB 3rd, Mark EJ, Kradin RL. Pulmonary "inflammatory myofibroblastic" tumors: a critical examination of the diagnostic category based on quantitative immunohistochemical analysis. Virchows Arch 2007;450:585-90.

24. Xing X, Feldman AL. Anaplastic large cell lymphomas: ALK positive, ALK negative, and primary cutaneous. Adv Anat Pathol 2015;22:29-49.

Cite this article as: Zhu T, Gao P, Gao J, Liu X, Zhang X. A huge recurrent retroperitoneal inflammatory myofibroblastic tumor implicating renal and ureter: a case report. Transl Cancer Res 2021;10(10):4565-4570. doi: 10.21037/tcr-21-899
25. Surabhi VR, Chua S, Patel RP, et al. Inflammatory Myofibroblastic Tumors: Current Update. Radiol Clin North Am 2016;54:553-63.

26. Chavez C, Hoffman MA. Complete remission of ALK-negative plasma cell granuloma (inflammatory myofibroblastic tumor) of the lung induced by celecoxib: A case report and review of the literature. Oncol Lett 2013;5:1672-6.

27. Chen M, Zhang L, Cao G, et al. Partial response to chemotherapy in a patient with retroperitoneal inflammatory myofibroblastic tumor. Mol Clin Oncol 2016;5:463-6. 